UDC 517.9

\title{
COMPLICATED DYNAMICS OF AN INTERACTING ECONOMIC MODEL
}

\section{СКЛАДНА ДИНАМІКА ВЗАЕМОДІЮЧОЇ ЕКОНОМІЧНОЇ МОДЕЛІ}

\author{
O. Stepanenko, Yu. Maistrenko
}

Inst. Math. Nat. Acad. Sci. Ukraine

Tereshchenkivs'ka Str., 3, Kyiv, 01601, Ukraine

Sh. Yousefi

SDU-Univ. Southern, Denmark

We study a $N$-dimensional dynamical system that presents interacting economic models, the so-called quantity setting oligopoly. Parameter regions of stability of the Nash equilibrium are obtained and the phase diagram for stability of the synchronized motion are investigated.

We also consider the problem of cluster formation. In particular, the 2-cluster state is studied extensively. A locally stable Nash equilibrium and quasiperiodic motions are present in this state. The regions of stability and the bifurcations of stable trajectories are investigated and corresponding bifurcation diagram are obtained.

Вивчається $N$-вимірна динамічна система, яка задає взаємодіючі економічні моделі і є так званою кількісною визначаючою олігополлю. Отримано області параметрів стійкості рівноважного стану Неша та вивчено фазову діаграму стійкості синхронного руху. Також розглянуто проблему утворення кластерів, зокрема, детально вивчено стан з двома кластерами. Цей стан має локально стійкий рівноважний стан Неша, а також має місце квазіперіодичний рух. Досліджено області стійкості і біфуркації стійких траєкторій і отримано відповідну біфуркаційну діаграму.

1. Introduction. Model. The Cournot model of imperfect competition between economic agents is a well-known concept in mathematical economics [1-3]. This model elaborates on a particular scenario when the market structure departs from monopoly towards a competitive framework. The simple case of the Cournot duopoly involves two agents competing for the lion share of the market. The more complicated case of the Cournot oligopoly involves an increasing number of competing agents who are able to collectively exert control over the supply of a given commodity. In all these cases, it is assumed that the expectations on the competitor's actions can be used to form the agent's own preferred action, which is profit-maximizing choice given by these expectations.

In the present study, a simple framework of the models previously studied in [4] and [5] is extended to the general case of oligopoly competition among a large number of agents in the market. A quantity-setting oligopoly where the agents provide a homogeneous good is considered. Despite the fairly restrictive assumption of the model, the obtained results appear to be useful in understanding the underlying dynamical properties of the general model. In doing so, the focus has been directed towards the mathematical description of the model followed by elaborations on possible economic interpretations of the obtained results.

(C) O. Stepanenko, Yu. Maistrenko, and Sh. Yousefi, 2004

ISSN 1562-3076. Нелінійні коливання, 2004, m. 7, №2 
The time evolution of a dynamic oligopoly game with $N$ competing firms is modelled by a discrete dynamical system obtained by the iteration of a $N$-dimensional noninvertible map. Output quantity of each firm is adjusted in response to the quantities observed in the previous period. Making the same assumptions as introduced in [5] and taking reaction functions in a form

$$
r_{i}=\mu_{i} \sum_{j \neq i} x_{j}\left(1-x_{j}\right), i=1, \ldots, N
$$

we have oligopoly game modelled by the map

$$
x_{i}^{(n+1)}=(1-\lambda) x_{i}^{(n)}+\lambda \mu \sum_{j \neq i} f\left(x_{j}^{(n)}\right),
$$

where $x_{i}^{(n)}>0, i=1, \ldots, N$, is a quantity of good produced by the $i$-th firm at the $n$-th period of time, $\lambda \in[0 ; 1]$ represent the speed of the adjustment process for achieving optimal quantity producing, $\mu_{i}>0$ represent an extent of the interfirm externality incorporated in cost functions of the firms, $f$ is a quadratic map $f(x)=x(1-x)$.

Let's denote

$$
F:\left(\begin{array}{c}
x_{1}^{(n)} \\
x_{2}^{(n)} \\
\vdots \\
x_{N}^{(n)}
\end{array}\right) \rightarrow\left(\begin{array}{c}
x_{1}^{(n+1)} \\
x_{2}^{(n+1)} \\
\vdots \\
x_{N}^{(n+1)}
\end{array}\right)
$$

The Jacobian matrix of map (1) is

$$
D F=\left(\begin{array}{cccc}
1-\lambda & \lambda \mu f^{\prime}\left(x_{2}\right) & \ldots & \lambda \mu f^{\prime}\left(x_{N}\right) \\
\lambda \mu f^{\prime}\left(x_{1}\right) & 1-\lambda & \ldots & \lambda \mu f^{\prime}\left(x_{N}\right) \\
\vdots & \vdots & \ddots & \vdots \\
\lambda \mu f^{\prime}\left(x_{1}\right) & \lambda \mu f^{\prime}\left(x_{2}\right) & \ldots & 1-\lambda
\end{array}\right)
$$

Below in this study we assume $x_{i}>0, i=1, \ldots, N$.

2. Synchronized state. Concider a synchronized state, i. e., when dynamics of the map (1) is confined within the diagonal

$$
D=\left\{x_{1}=x_{2}=\ldots=x_{N} \equiv x\right\}
$$

and the map can be written in the form

$$
g: x \rightarrow(1-\lambda) x+(N-1) \lambda \mu f(x)
$$

For the oligopoly modelled, it means that all the firms produce the same quantity of goods. Map (2) depends on two parameters $\lambda, \mu$ and can be reduced to the logistic map $\tilde{f}=\tilde{a} \tilde{x}(1-\tilde{x})$, where

$$
\tilde{a}=\frac{(1-\lambda+(N-1) \lambda \mu)^{2}}{(N-1) \lambda \mu}
$$


by substitution

$$
x=\frac{(1-\lambda+(N-1) \lambda \mu)}{(N-1) \lambda \mu} \tilde{x} .
$$

Proposition 1. Eigenvalues of the Jacobian matrix DF of the map (1) in the diagonal $D$ are equal to

$$
\begin{aligned}
& \nu_{1}=1-\lambda+(N-1) \lambda \mu f^{\prime}(x), \\
& \nu_{2, \ldots, N}=1-\lambda-\lambda \mu f^{\prime}(x) .
\end{aligned}
$$

Proof. The characteristic equation for eigenvalues of the Jacobian matrix $D F$ in the diagonal $D$ assumes the form

$$
|D F|_{D}-\nu I|=| \begin{array}{cccc}
1-\lambda-\nu & \lambda \mu f^{\prime}(x) & \ldots & \lambda \mu f^{\prime}(x) \\
\lambda \mu f^{\prime}(x) & 1-\lambda-\nu & \ldots & \lambda \mu f^{\prime}(x) \\
\vdots & \vdots & \ddots & \vdots \\
\lambda \mu f^{\prime}(x) & \lambda \mu f^{\prime}(x) & \ldots & 1-\lambda-\nu
\end{array} \mid=0 .
$$

By matrix transformation, it is reduced to the equation

$$
\left(1-\lambda-\nu+(N-1) \lambda \mu f^{\prime}(x)\right)\left(1-\lambda-\nu-\lambda \mu f^{\prime}(x)\right)^{(N-1)}=0,
$$

which gives us solution (3).

Remark 1. For further consideration let's denote $\nu_{1}=: \nu_{\|}, \nu_{2, \ldots, N}=: \nu_{\perp}$, since $\nu_{1}$ corresponds to the eigenvector which is parallel to the diagonal and $\nu_{2, \ldots, N}$ corresponds to eigenvectors which are perpendicular to it.

2.1. Region of stability of the Nash equilibrium. Consider a nontrivial fixed point $x^{*}$ of the map which is situated on the diagonal $D(1)$ (It is the so-called symmetric Nash equilibria for the oligopoly.) We can find it by solving the equation

$$
(1-\lambda) x+(N-1) \lambda \mu x(1-x)=x .
$$

Then

$$
x^{*}=1-\frac{1}{(N-1) \mu} .
$$

Eigenvalues of the Jacobi matrix in the fixed point are equal to

$$
\begin{aligned}
& \left.\nu_{\|}\right|_{x=x^{*}}=1+\lambda-(N-1) \lambda \mu, \\
& \left.\nu_{\perp}\right|_{x=x^{*}}=1-\lambda-\lambda\left(\frac{2}{N-1}-\mu\right) .
\end{aligned}
$$

The region of stability of $x^{*}$ in the parameters space $(\lambda, \mu)$ is bounded by the curves

$$
\mu_{1}=\frac{1}{N-1},\left[\nu_{\|}=1\right], \quad \lambda_{2}=\frac{2}{(N-1) \mu-1},\left[\nu_{\|}=-1\right], \quad \mu_{3}=1+\frac{2}{N-1},\left[\nu_{\perp}=1\right] .
$$

The region is represented in Fig. $1\left(\right.$ marked as $P_{1}^{(s)}$ ) for different $N$. 

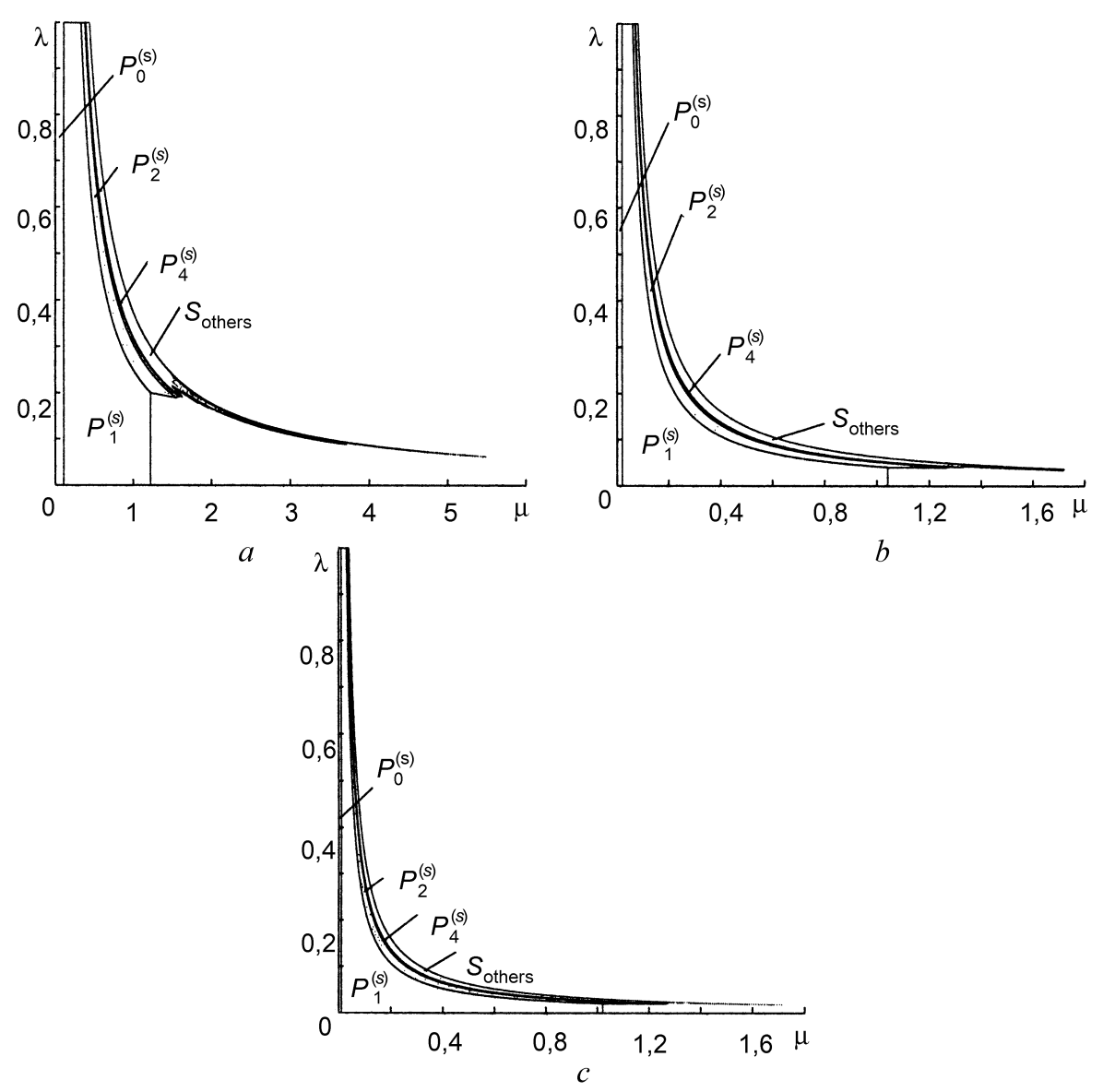

Fig. 1. Bifurcation diagram of the behavior on the diagonal for $N=10(a), N=50(b), N=$ $=100(c) \cdot P_{0}^{(s)}$ represents the region of stability of the trivial fixed point $(0, \ldots, 0) ; P_{1}^{(s)}$ represents the region of stability of a fixed point in the diagonal; $P_{2}^{(s)}$ represents the region of stability of period 2 orbit; $P_{4}^{(s)}$ represents the region of stability of period 4 orbit; $S_{\text {others }}$ represents the region where the trajectories in the diagonal of the period $>4$ and those that have chaotic motion are transversally stable.

2.2. Bifurcation scenario in the diagonal. Regions of stability of period 2 and period 4 cycles and some other cycles which lie on the diagonal are shown in Fig. 1 ( denoted by $P_{2}^{(s)}, P_{4}^{(s)}$, $\left.S_{\text {others }}\right)$.

On the line $\mu_{1}=\frac{1}{N-1}$, a transcritical bifurcation occurs when the trivial fixed point $x_{0}=0$ loses its stability, and the nontrivial stable fixed point $x^{*}=1-\frac{1}{(N-1) \mu}$, appears. On the curve $\lambda_{2}=\frac{2}{(N-1) \mu-1}$, there occurs a supercritical period doubling bifurcation (the eigenvalue $\left.\nu_{\|}=-1\right)$. The fixed point $x^{*}=1-\frac{1}{(N-1) \mu}$ loses its stability and a stable period 2 cycle appears.

As $\lambda$ and $\mu$ increase, new stability regions in the diagonal appear. The order of appearance of these regions corresponds to periodic windows of the logistic map (on the line $\lambda=1$ the 


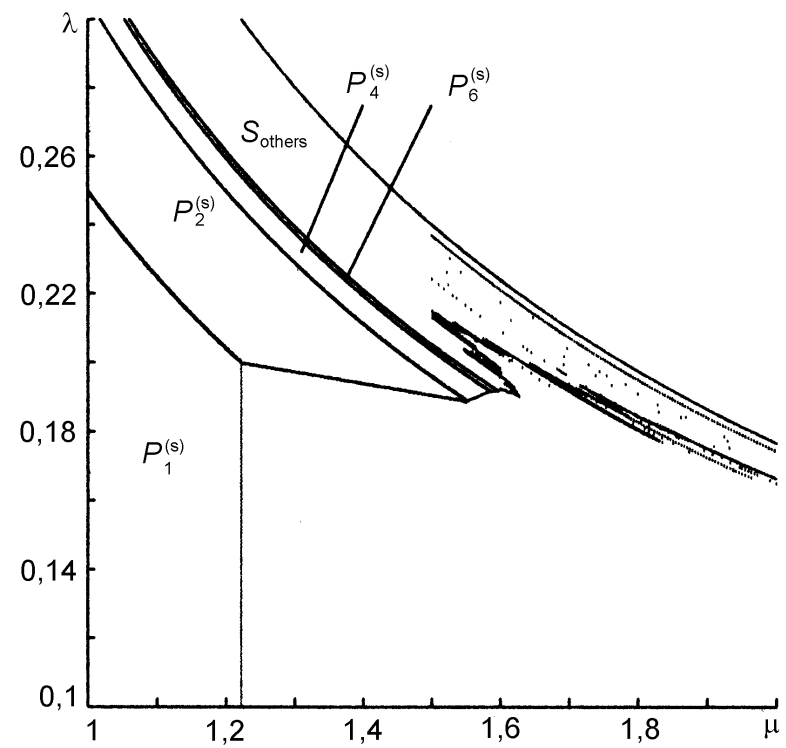

Fig. 2. A more detailed picture of the bifurcation diagram on the diagonal for $N=10$. It shows a period doubling order of the appearance of stable trajectories with a synchronized periodic motion.

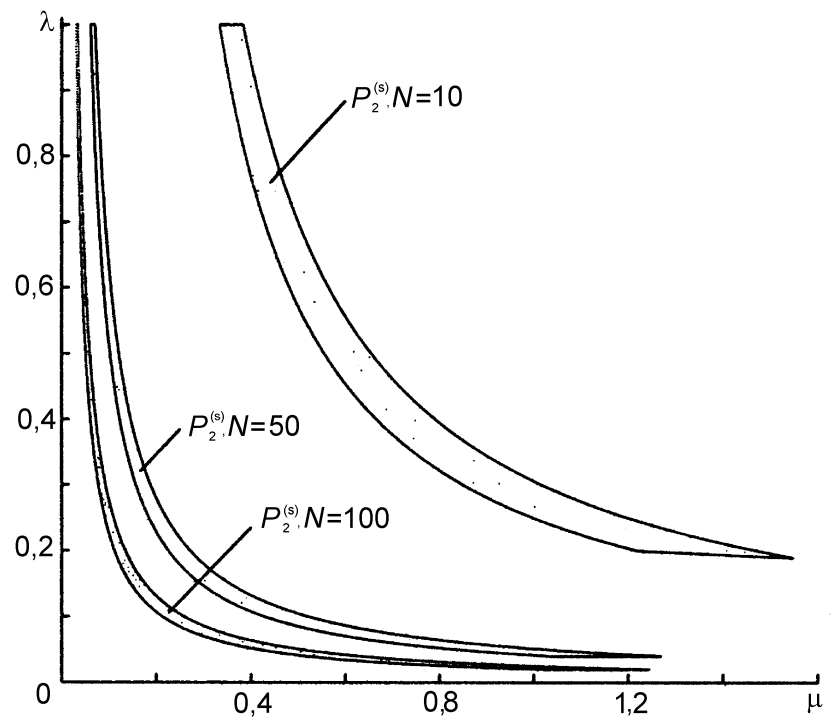

Fig. 3. Regions of stability of a period 2 cycle in the diagonal, with $N=10,50$, and 100 , respectively.

map $g$ completely coincides with the canonical form of the logistic map $a x(1-x)$ ). Fig. 2 shows a more deteiled picture of the bifurcation diagram on the diagonal for $N=10$.

Dependence of the regions' sizes on the system size $N$ is represented in Fig. 3. Thus, the regions are strongly decreasing as $N$ increases. 
3. Stability of the 2-cluster state. Consider a 2-cluster state that arises when all coordinates $x_{i}$ are divided into two groups (clusters) with the dynamics being identical in each group. Thus the total dynamics is confined to the 2-dimension manifold

$$
D_{2}:\left\{\begin{array}{l}
x_{1}=x_{2}=\ldots=x_{N_{1}} \\
x_{N_{1}+1}=\ldots=x_{N}
\end{array}\right.
$$

where $N_{1}$ is the number of elements in the first group. Denote $N_{2}:=N-N_{1}, x:=x_{1}=\ldots$ $\ldots=x_{N_{1}}, y:=x_{N_{1}+1}=\ldots=x_{N}$. Then, the dynamics of the map is described by the 2-dimension map

$$
G_{2}:\left(\begin{array}{l}
x \\
y
\end{array}\right) \rightarrow\left(\begin{array}{c}
(1-\lambda) x+\lambda \mu\left(N_{1}-1\right) f(x)+\lambda \mu N_{2} f(y) \\
(1-\lambda) y+\lambda \mu N_{1} f(x)+\lambda \mu\left(N_{2}-1\right) f(y)
\end{array}\right) .
$$

To investigate the behaviour of map (1) in the manifold $D_{2}$, first, "in-cluster" eigenvalues $\nu_{1,2}$ should be found. We call them "in-cluster" since they describe the internal dynamics of the 2-clusters developed in the manifold $D_{2}$, that is, the behaviour of groups of firms rather than individual firms. So, we solve the characteristic equation

$$
\left|D G_{2}-\nu I\right|=\left|\begin{array}{cc}
1-\lambda-\nu+\lambda \mu\left(N_{1}-1\right) f^{\prime}(x) & \lambda \mu N_{2} f^{\prime}(y) \\
\lambda \mu N_{1} f^{\prime}(x) & 1-\lambda-\nu+\lambda \mu\left(N_{2}-1\right) f^{\prime}(x)
\end{array}\right|=0 .
$$

The eigenvalues are found to be

$$
\begin{aligned}
\nu_{1,2}= & -\lambda+\frac{\lambda \mu}{2}\left(\left(N_{1}-1\right) f^{\prime}(x)+\left(N_{2}-1\right) f^{\prime}(y) \pm\right. \\
& \left. \pm \sqrt{\left(\left(N_{1}-1\right) f^{\prime}(x)-\left(N_{2}-1\right) f^{\prime}(y)\right)^{2}+4 N_{1} N_{2} f^{\prime}(x) f^{\prime}(y)}\right)
\end{aligned}
$$

To evaluate the transversal stability of the 2-cluster state in the whole $N$-dimensional space, we need to find all other transversal eigenvalues. They are found from the characteristic equation of the form

$$
|D F|_{D_{2}}-\nu I|=| \begin{array}{ccccccc}
1-\lambda-\nu & \lambda \mu f^{\prime}(x) & \ldots & \lambda \mu f^{\prime}(x) & \lambda \mu f^{\prime}(y) & \ldots & \lambda \mu f^{\prime}(y) \\
\lambda \mu f^{\prime}(x) & 1-\lambda-\nu & \ldots & \lambda \mu f^{\prime}(x) & \lambda \mu f^{\prime}(y) & \ldots & \lambda \mu f^{\prime}(y) \\
\vdots & \vdots & \ddots & \vdots & \vdots & \vdots & \vdots \\
\lambda \mu f^{\prime}(x) & \lambda \mu f^{\prime}(x) & \ldots & 1-\lambda-\nu & \lambda \mu f^{\prime}(y) & \ldots & \lambda \mu f^{\prime}(y) \\
\lambda \mu f^{\prime}(x) & \lambda \mu f^{\prime}(x) & \ldots & \lambda \mu f^{\prime}(x) & 1-\lambda-\nu & \ldots & \lambda \mu f^{\prime}(y) \\
\vdots & \vdots & \vdots & \vdots & \vdots & \ddots & \vdots \\
\lambda \mu f^{\prime}(x) & \lambda \mu f^{\prime}(x) & \ldots & \lambda \mu f^{\prime}(x) & \lambda \mu f^{\prime}(y) & \ldots & 1-\lambda-\nu
\end{array} \mid=0 .
$$

By using matrix transformations, it can be reduced to the form

$$
\begin{gathered}
\left|\begin{array}{cc}
1-\lambda-\nu+\left(N_{1}-1\right) \lambda \mu f^{\prime}(x) & N_{2} \lambda \mu f^{\prime}(y) \\
N_{1} \lambda \mu f^{\prime}(x) & 1-\lambda-\nu+\left(N_{2}-1\right) \lambda \mu f^{\prime}(y)
\end{array}\right| \times \\
\quad \times\left(1-\lambda-\nu-\lambda \mu f^{\prime}(x)\right)^{N_{1}-1}\left(1-\lambda-\nu-\lambda \mu f^{\prime}(y)\right)^{N_{2}-1}=0 .
\end{gathered}
$$



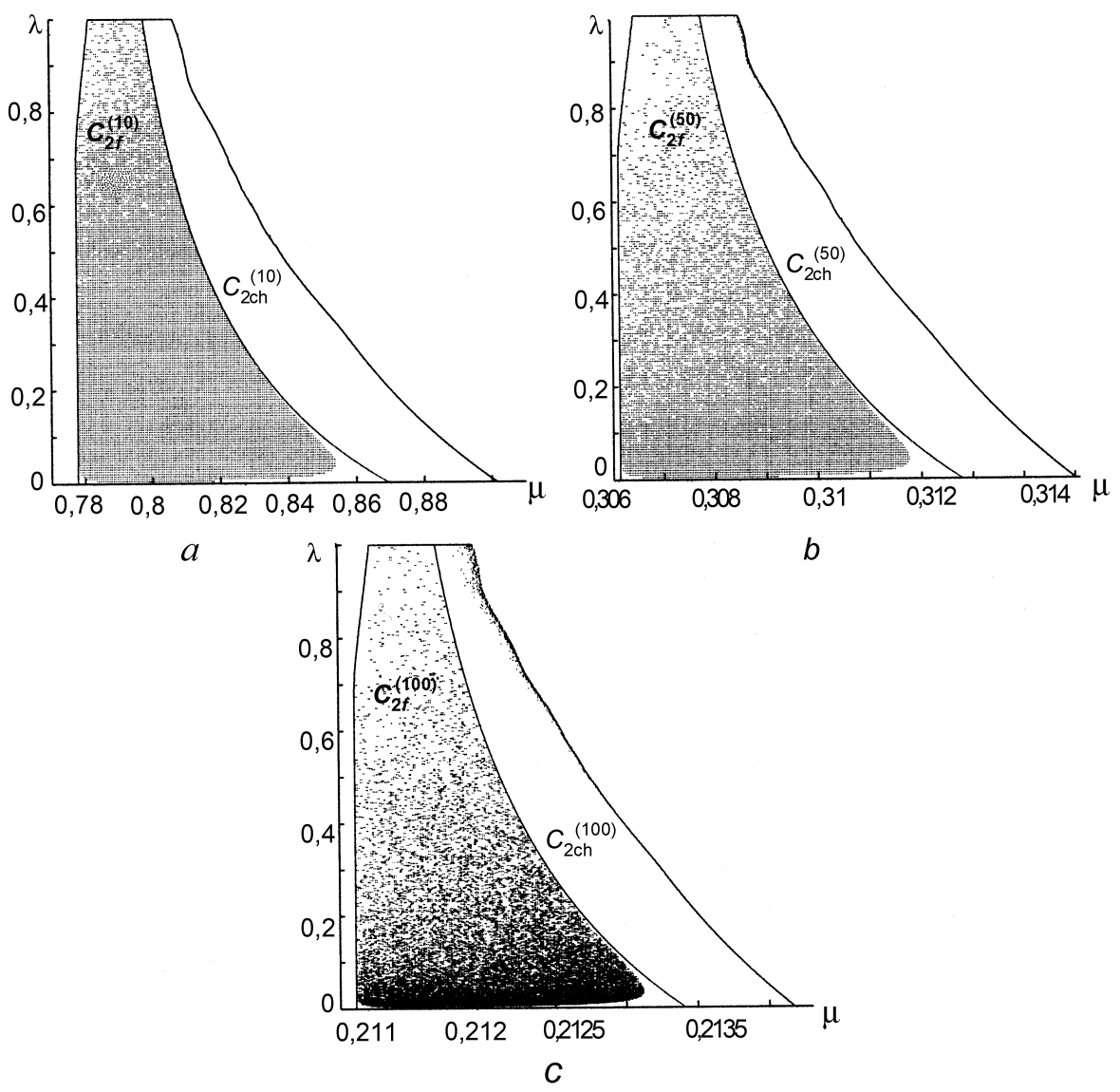

C

Fig. 4. Stability regions of the fixed points (labeled by $C_{f}^{\left(1: N_{2}\right)}$ ) and quasiperiodic attractors (labeled by $C_{c h}^{\left(1: N_{2}\right)}$ ) if the cluster ratio $N_{1}: N_{2}$ is $1: 9(a), 1: 49(b), 1: 99(c)$.

Thus, there are two different transversal eigenvalues:

$$
\begin{aligned}
& \nu_{\perp, 1}:=\nu_{3, \ldots, N_{1}+1}=1-\lambda-\lambda \mu f^{\prime}(x), \\
& \nu_{\perp, 2}:=\nu_{N_{1}+2, \ldots, N}=1-\lambda-\lambda \mu f^{\prime}(y) .
\end{aligned}
$$

These eigenvalues determine stability regions of the 2-cluster state and conditions for its destruction. Moreover, if $\left|\nu_{3, N_{1}+1}\right|>1$ the first sub-cluster is splited and if $\left|\nu_{N_{1}+2, N}\right|>1$ the second sub-cluster is splited before the destruction.

3.1. Fixed points out of the diagonal. The fixed points can be found from the equations:

$$
\begin{aligned}
& x=(1-\lambda) x+\left(N_{1}-1\right) \lambda \mu x(1-x)+N_{2} \lambda \mu y(1-y), \\
& y=(1-\lambda) y+N_{1} \lambda \mu x(1-x)+\left(N_{2}-1\right) \lambda \mu y(1-y) .
\end{aligned}
$$

By solving the system we get a cubic equation that can be easily solved numericaly. In the parameters range under consideration this equation has three real roots which provide three fixed points of the map (4), one is on the diagonal $D$ and two others outside of it.

Fig. 4 shows regions of stability of fixed points of clusters if the ratio $N_{1}: N_{2}$ is $1: 9$, 1 : 49, 1 : 99. Solid lines outline regions obtained by using an analytical method, and the 


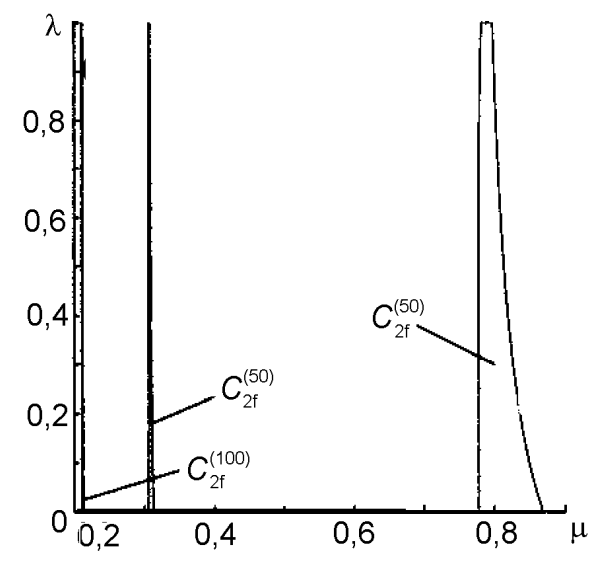

Fig. 5. A comparison of sizes of the regions of the stability fixed points in clusters if the ratio $N_{1}: N_{2}$ is $1: 9,1: 49,1: 99$.

points inside the regions are calculated in a numerical simulation. The density of the points indirectly shows the dependence of the size of the basin of attraction of fixed points on the parameter values. The higher the density, the larger region and the higher the probability for the trajectory with randomly chosen initial conditions to be attracted to the fixed point. Fig. 5 shows a dependence of sizes of the regions of the stability of fixed points in clusters if the ratio $N_{1}: N_{2}$ is $1: 9,1: 49,1: 99$ on the dimensions of the system $(N=10,50,100)$.

1. Топи Рии Attractors, bifurcations, and chaos. Nonlinear phenomena in economics. - Berlin etc.: Springer, 2000. - $507 \mathrm{p}$.

2. Rand D. Exotic phenomena in games and duopoly models // J. Math. Econ. - 1978. - 5 . - P. $173-178$.

3. Ahmed E., Agiza H. N. Dynamics of a Cournot game with $n$ competitors // Chaos, Solutions and Fractals. 1998. - 9. - P. $1513-1517$.

4. Kopel M. Simple and complex adjustment dynamics in Cournot duopoly models // Ibid. - 1996. - 7. P. $2031-2048$.

5. Agiza H. N., Bischi G. I., and Kopel M. Multistability in a dynamic Cournot game with three oligopolists // Math. and Comput. Simulat. - 1999. - 51. - P. $63-90$.

Received 02.07.2003 